\title{
A SPECTROSCOPIC REDSHIFT MEASUREMENT FOR A LUMINOUS LYMAN BREAK GALAXY AT $z=7.730$ USING KECK/MOSFIRE
}

\author{
P. A. Oesch ${ }^{1,2}$, P. G. van Dokkum ${ }^{2}$, G. D. Illingworth ${ }^{3}$, R. J. Bouwens ${ }^{4}$, I. Momcheva ${ }^{2}$, B. Holden $^{3}$, \\ G. W. Roberts-Borsani ${ }^{4,5}$, R. Smit ${ }^{6}$, M. Franx ${ }^{4}$, I. Labbé ${ }^{4}$, V. González ${ }^{7}$, and D. MageE ${ }^{3}$ \\ ${ }^{1}$ Yale Center for Astronomy and Astrophysics, Physics Department, New Haven, CT 06520, USA; pascal.oesch@yale.edu \\ ${ }^{2}$ Department of Astronomy, Yale University, New Haven, CT 06520, USA \\ ${ }^{3}$ UCO/Lick Observatory, University of California, Santa Cruz, CA 95064, USA \\ ${ }^{4}$ Leiden Observatory, Leiden University, NL-2300 RA Leiden, The Netherlands \\ ${ }^{5}$ Department of Physics and Astronomy, University College London, Gower Street, London WC1E 6BT, UK \\ ${ }^{6}$ Centre for Extragalactic Astronomy, Department of Physics, Durham University, South Road, Durham DH1 3LE, UK \\ ${ }^{7}$ University of California, Riverside, 900 University Ave., Riverside, CA 92507, USA \\ Received 2015 February 18; accepted 2015 April 9; published 2015 May 5
}

\begin{abstract}
We present a spectroscopic redshift measurement of a very bright Lyman break galaxy at $z=7.7302 \pm 0.0006$ using the Keck/Multi-Object Spectrometer for Infra-Red Exploration. The source was pre-selected photometrically in the EGS field as a robust $z \sim 8$ candidate with $H=25.0$ mag based on optical non-detections and a very red Spitzer/IRAC [3.6]-[4.5] broad-band color driven by high equivalent width [O III] $+\mathrm{H} \beta$ line emission. The Ly $\alpha$ line is reliably detected at $6.1 \sigma$ and shows an asymmetric profile as expected for a galaxy embedded in a relatively neutral intergalactic medium near the Planck peak of cosmic reionization. The line has a rest-frame equivalent width of $\mathrm{EW}_{0}=21 \pm 4 \AA$ and is extended with $V_{\text {FWHM }}=360_{-70}^{+90} \mathrm{~km} \mathrm{~s}^{-1}$. The source is perhaps the brightest and most massive $z \sim 8$ Lyman break galaxy in the full CANDELS and BoRG/HIPPIES surveys, having already assembled $10^{9.9 \pm 0.2} M_{\odot}$ of stars at only $650 \mathrm{Myr}$ after the Big Bang. The spectroscopic redshift measurement sets a new redshift record for galaxies. This enables reliable constraints on the stellar mass, star formation rate, and formation epoch, as well as combined $[\mathrm{O}$ III $]+\mathrm{H} \beta$ line equivalent widths. The redshift confirms that the IRAC [4.5] photometry is very likely dominated by line emission with $\mathrm{EW}_{0}\left(\left[\mathrm{O}_{\mathrm{III}}\right]+\mathrm{H} \beta\right)=720_{-150}^{+180} \AA$. This detection thus adds to the evidence that extreme rest-frame optical emission lines are a ubiquitous feature of early galaxies promising very efficient spectroscopic follow-up in the future with infrared spectroscopy using the James Webb Space Telescope and, later, ELTs.
\end{abstract}

Key words: dark ages, reionization, first stars - galaxies: evolution - galaxies: formation - galaxies: high-redshift

\section{INTRODUCTION}

The spectroscopic confirmation and characterization of galaxy candidates within the cosmic reionization epoch has been a major challenge for observational extragalactic astronomy for the last few years. Recently, large samples of several hundred galaxy candidates have been identified at $z$ $\sim 7-11$ thanks to the exceptional near-infrared sensitivity of the WFC3/IR camera on board the Hubble Space Telescope (HST; e.g., Bouwens et al. 2011, 2015; Oesch et al. 2012, 2014; McLure et al. 2013; Schenker et al. 2013; Finkelstein et al. 2015). However, despite this unprecedented target sample, very little progress has been made in spectroscopically confirming galaxies in the cosmic reionization epoch. Currently, only a handful of normal galaxies have reliably measured redshifts at $z>7$ (see, e.g., Pentericci et al. 2011; Vanzella et al. 2011; Ono et al. 2012; Schenker et al. 2012; Shibuya et al. 2012; Finkelstein et al. 2013), with most spectroscopic surveys being unsuccessful or only resulting in uncertain candidate lines (e.g., Jiang et al. 2013a; Treu et al. 2013; Tilvi et al. 2014; Caruana et al. 2014; Faisst et al. 2014; Vanzella et al. 2014; Schenker et al. 2014).

Current studies at $z>6$ thus rely on photometric samples, with selection criteria and photometric redshifts that are somewhat uncertain due to the difficulty of establishing reliable priors of potential contaminant populations at lower redshift. Spectroscopic follow-up is therefore particularly important for the very rare galaxies at the bright end of the UV luminosity and mass functions where any contamination has a very large impact.

The low success rate of spectroscopic follow-up surveys is likely caused by a decreased fraction of galaxies showing strong Ly $\alpha$ emission due to an increased neutral fraction in the intergalactic medium (IGM) at $z>6$ (Stark et al. 2010, 2011; Schenker et al. 2012, 2014; Treu et al. 2013; Pentericci et al. 2014). While Ly $\alpha$ is the primary spectral feature for spectroscopic confirmation of high-redshift candidates, new surveys targeting the Lyman continuum break are underway using the WFC3/IR grism on the HST, or alternatively, weak UV lines may also be detectable from the ground (Stark et al. 2015).

Two recent successful Ly $\alpha$ detections of Lyman break selected galaxies at $z>7.2$ were published in Ono et al. (2012, $z=7.213)$ and Finkelstein et al. $(2013, z=7.508)$. Both these sources are relatively bright with $H=25.2$ and 25.6 mag and both show a significant flux excess in their IRAC photometry, which is consistent with extremely strong [O $\mathrm{III}]$ line emission at $4.1-4.3 \mu \mathrm{m}$. Such strong lines are characteristic of early starforming galaxies, as evidenced by a clear increase in broadband flux excess with redshift (e.g., Schaerer \& de Barros 2009; Labbé et al. 2013; Stark et al. 2013; González et al. 2014; Smit et al. 2014). Such excesses can be used to select relatively clean samples of star-forming galaxies at $z \sim 6.6-6.9$ as well as $z \sim 8$ (e.g., Smit et al. 2015, G. W. Roberts-Borsani et al. 2015, in preparation). 
In this Letter we present a successful spectroscopic redshift measurement at $z \sim 8$ using the Keck/Multi-Object Spectrometer for Infra-Red Exploration (MOSFIRE) of one of the brightest Lyman break galaxies (LBGs) at that epoch. This galaxy was pre-selected as a high-priority target because of a very red [3.6]-[4.5] color, likely caused by strong [O $\mathrm{III}]$ emission. Our target selection is summarized in Section 2, while Section 3 outlines the spectroscopic observations, and our results are presented in Section 4.

Throughout this paper, we adopt $\Omega_{M}=0.3, \Omega_{\Lambda}=0.7$, $H_{0}=70 \mathrm{kms}^{-1} \mathrm{Mpc}^{-1}$, i.e., $h=0.7$, consistent with the measurements from Planck (Planck Collaboration et al. 2015). Magnitudes are given in the AB system.

\section{TARGET SELECTION}

We briefly summarize our selection of a robust $z \sim 8 \mathrm{LBG}$ sample over the CANDELS fields using extreme IRAC photometry. For more details see G. W. Roberts-Borsani et al. (2015, in preparation).

The selection builds on that of Smit et al. (2015), who identify a sample of $z \sim 6.8$ galaxies based on strong [O $\mathrm{III}]$ $\lambda \lambda 4959,5007$ plus $\mathrm{H} \beta$ emission lines resulting in very blue [3.6]-[4.5] IRAC colors. As these lines shift into the IRAC 4.5 $\mu \mathrm{m}$ band, galaxies at $z \sim 7$ to $z \sim 9$ exhibit red [3.6]-[4.5] IRAC colors (see also Labbé et al. 2013; Stark et al. 2013; Bowler et al. 2014).

We exploit the availability of deep Spitzer/IRAC photometry over the HST CANDELS-Wide fields to systematically search for bright galaxies with IRAC colors of [3.6]-[4.5]>0.5 mag in addition to a $\mathrm{Ly} \alpha$ break (i.e., a non-detection at $<1 \mu \mathrm{m}$ ), characteristic for $z>7$ galaxies. This resulted in two candidates with $H<25.1 \mathrm{mag}$ in the EGS field (see G. W. Roberts-Borsani et al. 2015, in preparation). Fortuitously, these two sources are $<6^{\prime}$ from each other and can be targeted in a single MOSFIRE mask.

Stamps and spectral energy distribution (SED) fits for one of these sources (EGS-zs8-1) are shown in Figure 1. The F606W, F814W, F125W, and F160W images come from the CANDELS survey (Grogin et al. 2011), while the IRAC images are from the SEDS survey (Ashby et al. 2013). Also shown are WFC3/IR F105W observations that are fortuitously available over this source as a result of a separate follow-up program (GO:13792, PI: Bouwens). Despite its modest depth, this $Y_{105}$ image still provides a highly improved photometric redshift measurement by constraining the spectral break at $1 \mu \mathrm{m}$.

As seen from the figure, the source EGS-zs8-1 is only detected at $>1 \mu \mathrm{m}$ in the WFC3/IR imaging as well as in both IRAC 3.6 and $4.5 \mu \mathrm{m}$ bands. The [3.6]-[4.5] color of this source is measured to be $0.53 \pm 0.09$, i.e., at the edge of our IRAC color selection window ([3.6]-[4.5] > 0.5).

\section{OBSERVATIONS}

\subsection{MOSFIRE Spectroscopy}

We use MOSFIRE (McLean et al. 2012) on the Keck I telescope for Y-band spectroscopy of our primary $z \sim 8$ targets in the search for their Ly $\alpha$ emission lines. MOSFIRE offers efficient multiplex observations over a field of view of $\sim 6^{\prime} \times 3^{\prime}$ at a spectral resolution of $R \sim 3000(R=3500$ and $R=2850$ for a 0.7 or 0.9 slit, respectively).

Data over the EGS field were taken during three nights, 2014 April 18, 23, and 25. While the first night was essentially lost

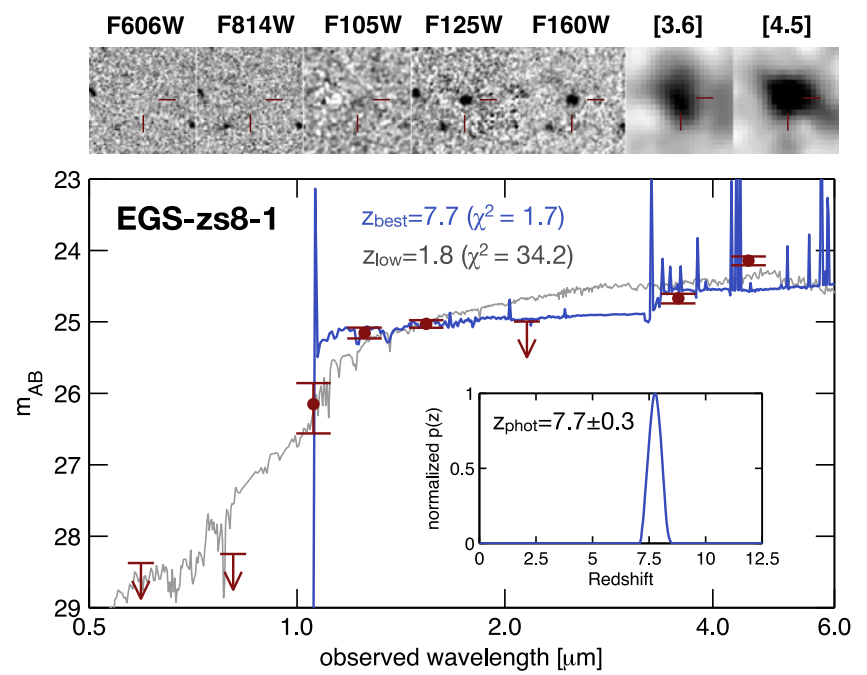

Figure 1. Top -images showing a $5^{\prime \prime} \times 5^{\prime \prime}$ region around our primary target galaxy EGS-zs8-1 in the HST and Spitzer/IRAC filters. These are, from left to right, $V_{606}, I_{814}, Y_{105}, J_{125}, H_{160}$, and $3.6 \mu \mathrm{m}$ and $4.5 \mu \mathrm{m}$. Bottom-the spectral energy distribution of EGS-zs8-1 based on fits to the HST+Spitzer+Kband photometry. Downward pointing vectors represent $2 \sigma$ upper limits in nondetection bands. A significant flux excess in the IRAC $4.5 \mu \mathrm{m}$ band is evident. Together with the strong spectral break, this constrains the photometric redshift to $z_{\text {phot }}=7.7 \pm 0.3$, in excellent agreement with the spectroscopic measurement as shown later. The best fit low-redshift solution at $z \sim 1.8$ is shown as a gray line for completeness. However, this SED has a likelihood of $<10^{-7}$ and is ruled out by the photometry.

due to bad seeing and clouds, the remaining nights had better conditions with a median seeing of $1^{\prime \prime}$ and only a few cirrus clouds during the last night. A dome shutter break problem also led to some vignetting during the last 30 minutes of the April 25 night before we stopped observations early. In total, we obtained $2.0 \mathrm{hr}$ of good quality Y-band spectroscopy during April 23 (Night 1) and $2.0 \mathrm{hr}$ on April 25 (Night 2).

Data were taken with $180 \mathrm{~s}$ exposures and $\mathrm{AB}$ dither offsets along the slit with $\pm 1^{\prime \prime}$ and \pm 1 ". 2 , respectively. In night 2 , we also increased the slit width from 0.7 (as used in Night 1) to 0.9 in anticipation of the slightly worse seeing forecast. During these nights we observed two masks with a total of eight $z \sim 7-8$ candidate galaxies in addition to lower redshift fillers.

\subsection{Data Reduction}

The data were reduced using a modified version of the public MOSFIRE reduction code DRP. ${ }^{8}$ This pipeline produces 2D sky-subtracted, rectified, and wavelength-calibrated data for each slitlet with a spatial resolution of 0.1799 per pixel and a dispersion of $1.086 \AA$ per pixel.

Each of our mask contains one slitlet placed on a star for monitoring the sky transparency and seeing conditions of each exposure. We use this star to track the mask drift across the detector (see, e.g., Kriek et al. 2015), which we find to be \pm 1.5 pixels $( \pm 0.27)$ and \pm 1 pixel $\left( \pm 00^{\prime \prime} 18\right)$ during night 1 and 2 , respectively. We separately reduce different batches of the data (of 30-45 minutes duration) to limit any signal-to-noise ratio reduction caused by this drift, before shifting and stacking the data.

The masks for the two nights have different orientations (Figure 2). The two independent data sets of the primary target

\footnotetext{
https://code.google.com/p/mosfire/
} 

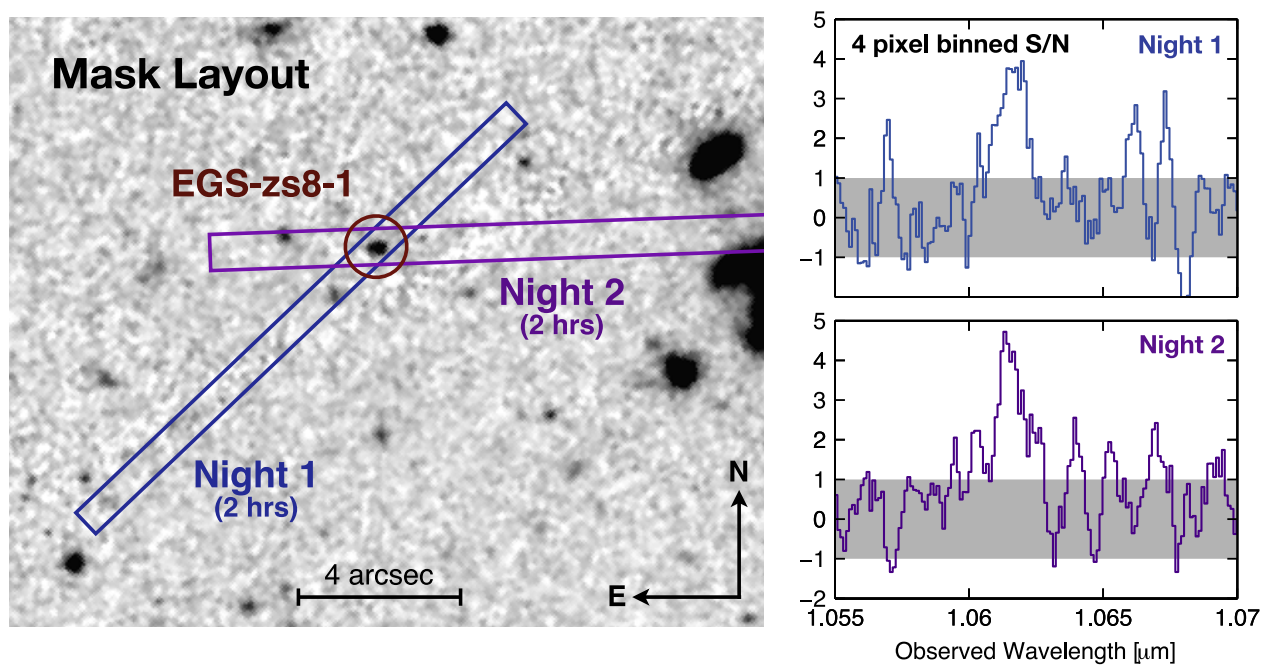

Figure 2. Left-mask layout of the two nights of MOSFIRE Y-band observations of our primary target. These two nights provide two completely independent measurements of this galaxy at two different orientations as well as two different positions along different slitlets. This also allows us to exclude the possibility of contamination in the final stacked spectrum from the two faint neighboring galaxies present within $2^{\prime \prime}$ of the primary galaxy along the slits. Right-the signal-to-noise ratio around the detected emission line in the two independent $1 \mathrm{D}$ spectra of the two nights, averaged over a 4 pixel width $(\sim 4 \AA)$. A line is clearly detected at $>4 \sigma$ independently in both $2 \mathrm{hr}$ spectra from each night. We also checked the unrectified frames to ensure that the positive flux in the spectrum indeed originated from the expected position of the galaxy along the spectrum.

thus add to the robustness of any detection. After creating the 2D spectra for the different masks, we applied the appropriate relative shift of the two $2 \mathrm{D}$ frames before stacking the observations of the two nights to our final 2D spectrum.

Similarly, 1D spectra were extracted separately for each mask using an optimal extraction based on a profile determined by the slit star. The extracted 1D spectra were corrected for Galactic extinction and for telluric absorption using nearby A0 stars observed in the same night at similar airmass. The uncertainty in our optimally extracted 1D spectra was determined empirically from empty rows in the full, rectified 2D spectra of the mask.

The absolute flux calibration was obtained from the slit stars by comparison of the spectra with the 3D-HST photometric catalogs (Skelton et al. 2014). An additional small correction was applied to account for the extension of individual sources in the slit mask by integrating the seeingmatched HST images over the slit and comparing with the slit loss of stellar sources.

\section{RESULTS}

Out of the eight $z \sim 7-8$ galaxy candidates, we detected a significant emission line $($ at $>5 \sigma)$ for only one source (EGSzs8-1). This line is discussed in detail below.

\subsection{A Ly $\alpha$ Emission Line at $z=7.730$}

The spectra of our target source EGS-zs8-1 (see Table 1 for summary of properties) revealed a significant emission line at the expected slit position in both masks independently (right panels Figure 2). The full $4 \mathrm{hr}$ stacked 2D and 1D spectra are shown in Figure 3, showing a line with a clear asymmetric profile, as expected for a $\operatorname{Ly} \alpha$ line at high redshift $(z \gtrsim 3)$. Furthermore, it lies at the expected wavelength based on our photometric redshift estimate $z_{\text {phot }}=7.7 \pm 0.3$. We therefore interpret this line as $\operatorname{Ly} \alpha$ (other possibilities are discussed in Section 4.3.
Table 1

Measurements of Galaxy EGS-zs8-1

\begin{tabular}{lc}
\hline \hline & Target \\
\hline R.A. (J2000) & $14: 20: 34.89$ \\
Decl. (J2000) & $53: 00: 15.4$ \\
$H_{160}$ & $25.03 \pm 0.05$ \\
$M_{U V}$ & $-22.06 \pm 0.05$ \\
\hline
\end{tabular}

\begin{tabular}{lc}
\hline \multicolumn{2}{c}{ Emission Line } \\
\hline$z_{\text {spec }}$ & \\
$f(\mathrm{Ly} \alpha)$ & $7.7302 \pm 0.0006$ \\
$L(\mathrm{Ly} \alpha)$ & $1.7 \pm 0.3 \times 10^{-17} \mathrm{erg} \mathrm{s}^{-1} \mathrm{~cm}^{-2}$ \\
$\mathrm{EW}_{0}(\mathrm{Ly} \alpha)^{\mathrm{a}}$ & $1.2 \pm 0.2 \times 10^{43} \mathrm{erg} \mathrm{s}^{-1}$ \\
$S_{w}$ & $21 \pm 4 \AA$ \\
FWHM $^{\mathrm{b}}$ & $15 \pm 6 \AA$ \\
$V_{\mathrm{FWHM}^{\mathrm{b}}}$ & $13 \pm 3 \AA$ \\
\end{tabular}

Physical Parameters $^{\mathrm{C}}$

\begin{tabular}{lc}
\hline $\log M_{\text {gal }} / M_{\odot}$ & $9.9 \pm 0.2$ \\
$\log$ age $\mathrm{yr}^{-1}$ & $8.0 \pm 0.5$ \\
$\log \mathrm{SFR} /\left(M_{\odot} \mathrm{yr}^{-1}\right)$ & $1.9 \pm 0.2$ \\
$\log \mathrm{SSFR}$ & $-8.0 \pm 0.4$ \\
$A_{\mathrm{UV}}$ & $1.6 \mathrm{mag}$ \\
$\mathrm{UV}$ slope $\beta$ & $-1.7 \pm 0.1$ \\
\hline
\end{tabular}

\footnotetext{
a Not corrected for IGM absorption.

b Derived from truncated Gaussian fit, corrected for instrumental broadening, but not for IGM absorption.

${ }^{\mathrm{c}}$ Based on SED fits (see Section 5; Oesch et al. 2014).
}

We fit the line using a Markov Chain Monte Carlo (MCMC) approach based on the emcee python library (ForemanMackey et al. 2013). Our model is based on a truncated Gaussian profile to account for the IGM absorption and includes the appropriate instrumental resolution. The model also includes the uncertainty on the background continuum level. The MCMC output provides full posterior PDFs and 


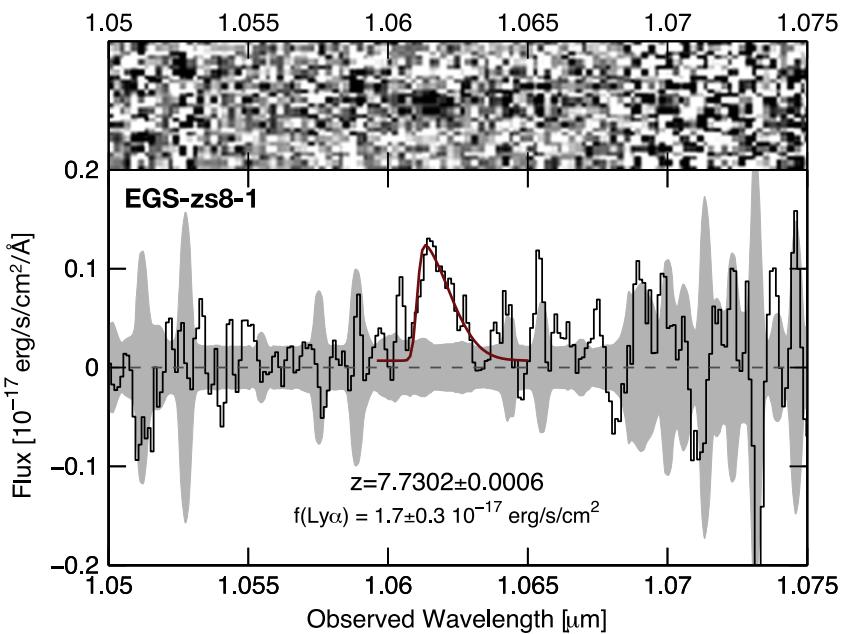

Figure 3. MOSFIRE spectra of EGS-zs8-1. The full 2D spectrum after two-bytwo binning is shown in the top panel, while the optimally extracted 1D spectrum is shown on the bottom. The 1D spectrum was smoothed by a 3 pixel ( $\sim 3 \AA)$ moving average filter for clarity. The gray shaded area represents the $1 \sigma$ flux uncertainty, while the dark red line shows the best-fit model. The line is quite extended in the wavelength direction and shows clear asymmetry with the expected shape typical for high-redshift Ly $\alpha$ lines. The spectroscopic redshift measurement is $z_{\text {spec }}=7.7302 \pm 0.0006$ in excellent agreement with the previously determined photometric redshift. Other line characteristics are summarized in Table 1.

uncertainties for the redshift, line flux, significance, and line width.

The line corresponds to a redshift of $z_{\mathrm{Ly} \alpha}=7.7302 \pm 0.0006$, with a total luminosity of $L_{\mathrm{Ly} \alpha}=1.2 \pm 0.2 \times 10^{43} \mathrm{erg} \mathrm{s}^{-1}$, and a total detection significance of $6.1 \sigma$. This is somewhat lower, but consistent with a simple estimate of $7.2 \sigma$ detection significance from integrating the 1D extracted pixel flux over the full extent of the line (i.e., not accounting for background continuum offsets).

Note that the redshift of the line is determined from our model of a truncated Gaussian profile and is thus corrected for instrumental resolution and the asymmetry arising from the IGM absorption. The peak of the observed line $(\lambda=10616 \AA)$ thus lies $\sim 2.5 \AA$ to the red of the actual determined redshift.

Given the brightness of the target galaxy, the detected line corresponds to a rest-frame equivalent width of $\mathrm{EW}_{0}=21 \pm 4$ $\AA$. This is lower than the $\mathrm{Ly} \alpha$ emitter criterion of $\mathrm{EW}_{0}>25 \AA$ set in recent analyses that use the Ly $\alpha$ fraction among LBGs to constrain the reionization process (e.g., Stark et al. 2011; Treu et al. 2013).

\subsection{Line Properties}

Different quantities of the detected line are tabulated in Table 1. In particular, we compute the weighted skewness parameter, $S_{w}$ (Kashikawa et al. 2006) finding $S_{w}=15 \pm 6 \AA$. This puts the line above the $3 \AA$ limit found for emission lines at lower redshift (see also Section 4.3).

The FWHM of the line is quite broad with $\mathrm{FWHM}=13 \pm 3 \AA$, corresponding to a velocity width of $V_{\text {FWHM }}=360_{-70}^{+90} \mathrm{~km} \mathrm{~s}^{-1}$. Our galaxy thus lies at the high end of the observed line width distribution for $z \sim 5.7-6.6$ Ly $\alpha$ emitters (e.g., Ouchi et al. 2010), but is consistent with previous $z>7$ Ly $\alpha$ lines (Ono et al. 2012).

\subsection{Caveats}

While the identification of the detected asymmetric emission line as Ly $\alpha$ is in excellent agreement with the expectation from the photometric redshift, we cannot rule out other potential identifications. As pointed out in the previous section, Kashikawa et al. (2006) find that weighted asymmetries $S_{w}>3 \AA$ are not seen in lower redshift lines, but almost exclusively in Ly $\alpha$ of high-redshift galaxies. However, at the resolution of our spectra, the observed asymmetry is also consistent with an $\left[\mathrm{O}_{\mathrm{II}}\right]$ line doublet in a high electron density environment, i.e., with a ratio of $\left[\mathrm{O}_{\text {II }}\right] \lambda 3726 /\left[\mathrm{O}_{\text {II }}\right] \lambda 3729>2$, and with a velocity dispersion of $\sigma_{v} \gtrsim 100 \mathrm{~km} \mathrm{~s}^{-1}$.

If the observed line is an $\left[\mathrm{O}_{\mathrm{II}}\right] \lambda \lambda 3726,3729$ doublet, the redshift of this galaxy would be $z_{\text {OII }}=1.85$. This is very close to the best low redshift SED fit shown in Figure 1. However, that SED requires a strong spectral break caused by an old stellar population, for which no emission line would be expected. Additionally, the low-redshift solution can not explain the extremely red IRAC color (used to select this galaxy), and predicts significant detections in the ACS/F814W band, as well as in the ground-based WIRDS K-band image (Bielby et al. 2012). No such detections are present, however, resulting in a likelihood for such an SED of $\mathcal{L}<10^{-7}$ (see also Figure 1). Thus all the evidence points to this line being Ly $\alpha$ at $z=7.73$.

\section{DISCUSSION}

In this Letter we used Keck/MOSFIRE to spectroscopically confirm the redshift of one of the brightest $z \sim 8$ galaxies identified by Bouwens et al. (2015) over the five CANDELS fields. Interestingly, this source is $\sim 0.5$ mag brighter than any source identified in the wide-area BoRG and HIPPIES surveys (e.g., Trenti et al. 2011; Yan et al. 2011; Bradley et al. 2012; Schmidt et al. 2014).

As shown in Figure 4, with $z_{\text {spec }}=7.730$ and an absolute magnitude $M_{\mathrm{UV}}=-22.06 \pm 0.05$ the source EGS-zs8-1 is currently the most distant and brightest spectroscopically confirmed galaxy (apart from a gamma-ray burst at $z=8.2$; Salvaterra et al. 2009; Tanvir et al. 2009). EGS-zs8-1 also populates the brightest bin of the recent Bouwens et al. (2015) $z \sim 8$ UV luminosity function (LF), which makes it an unusually rare object. A spectroscopic confirmation of its high redshift is thus particularly valuable for proving the existence of bright $H=25.0 \mathrm{mag}$ galaxies at $z \sim 8$ and for validating the bright end LF constraints.

An SED fit at the spectroscopic redshift of the source reveals a relatively high stellar mass $\log M / M_{\odot}=9.9 \pm 0.2$, a star formation rate $(\mathrm{SFR})$ of $\log \mathrm{SFR} /\left(M_{\odot} \mathrm{yr}^{-1}\right)=1.9 \pm 0.2$, and a relatively young, but not extreme age of log age $\mathrm{yr}^{-1}$ $=8.0 \pm 0.5$ based on an apparent Balmer break between the WFC3/IR and the Spitzer photometry (see also Table 1). The corresponding formation redshift of this galaxy thus lies at $z_{f}=8.8$. For details on our SED fitting see, e.g., Oesch et al. (2014).

Interestingly, the source has a UV continuum slope of $\beta=-1.7 \pm 0.1$ (measured from the SED fit) and is consistent with considerable dust extinction, $E(B-V)=0.15 \mathrm{mag}$. The detection of a significant Ly $\alpha$ emission line is not inconsistent, however, given the complexities of line formation in such young galaxies. 

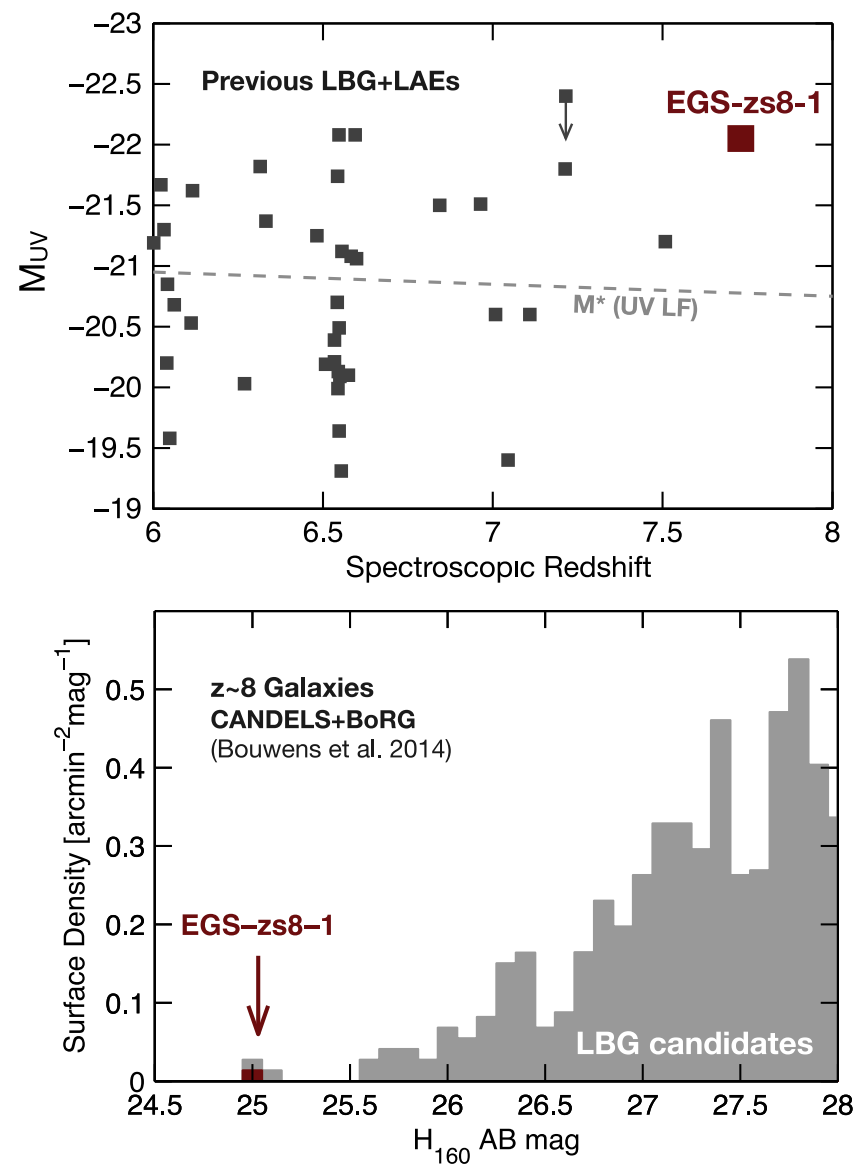

Figure 4. Top-UV absolute magnitudes of spectroscopically confirmed Lyman break galaxies and Ly $\alpha$ emitters in the cosmic reionization epoch, at $z>6$. Our target, EGS-zs8-1 (red square), represents the highest-redshift source and is the brightest galaxies currently confirmed. For reference, the gray dashed line shows the evolution of the characteristic magnitude $M_{*}$ of the UV LF (Bouwens et al. 2015). The galaxies shown as black squares are assembled from a compilation from Jiang et al. (2013b), Finkelstein et al. (2013), Shibuya et al. (2012), Ono et al. (2012), and Vanzella et al. (2011). Bottom-surface density of the full sample of $z \sim 8$ galaxies in the combined CANDELS and BoRG/HIPPIES fields (Bouwens et al. 2015, gray histogram). EGS-zs8-1 is the brightest and also one of the most massive sources at these redshifts. Note that all three $z \sim 8$ candidates with $H \sim 25.0$ mag $(\sim 0.5$ mag brighter than the rest) are identified in the CANDELS/WIDE survey area where ancillary $Y_{105}$ imaging is generally not available. Our spectroscopic confirmation is thus especially valuable.

These observations also allow us to reliably constrain the equivalent widths of the $[\mathrm{O}$ III] $]+\mathrm{H} \beta$ emission lines in this galaxy based on its IRAC colors. At the spectroscopic redshift of the source, these lines are shifted in the $4.5 \mu \mathrm{m}$ channel resulting in a color of [3.6]-[4.5] $=0.53 \pm 0.09$ mag. This is consistent with a combined rest-frame equivalent width of $\mathrm{EW}_{0}([\mathrm{O}$ III $]+\mathrm{H} \beta)=720_{-150}^{+180} \AA$.

Such a high equivalent width is in some tension with the inferred stellar population age of $\sim 100 \mathrm{Myr}$. However, it is completely consistent with the average $\mathrm{EW}_{0}([\mathrm{O} \mathrm{III}]+\mathrm{H} \beta)$ found for $z \sim 7-8$ galaxies in previous work (e.g., Labbé et al. 2013; Smit et al. 2014, 2015), where such strong lines were found to be ubiquitous (see also Laporte et al. 2014).

The fact that these strong lines are seen in a significant fraction of the $z \sim 7-8$ galaxies is at odds with the interpretation of extremely young galaxy ages of $<10 \mathrm{Myr}$ (e.g., Finkelstein et al. 2013). Nevertheless, very stochastic star formation may explain some of the line strength. Instead, it is possible that an evolution in the ionization properties of early galaxy populations may be causing stronger emission lines with more extreme line ratios $[\mathrm{O} \mathrm{III}] / \mathrm{H} \beta$ as is observed at $z \sim 3-4$ (see, e.g., Holden et al. 2015). We will discuss models that will provide greater insights into these strong emission line sources in a future paper.

Our confirmation of a source with extremely strong restframe optical emission lines at $z_{\text {spec }}=7.730$ together with two very similar sources at $z_{\text {spec }}=7.213$ and 7.508 (Ono et al. 2012; Finkelstein et al. 2013) provides further support for the likelihood of ubiquitous strong rest-frame optical lines as evidenced in the IRAC photometry of $z \sim 7-8$ galaxies. This has important consequences for future observations with the James Webb Space Telescope, which promises extremely efficient spectroscopic follow-up of such strong line emitters with NIRspec out to the highest redshifts of currently known galaxies.

The authors thank the referee, Eros Vanzella, for very helpful feedback that improved this paper. This work was supported by NASA grant NAG5-7697 and NASA grants HSTGO-11563.01 and HST-GO-13792. R.S. acknowledges the support of the Leverhulme Trust. The authors wish to recognize and acknowledge the very significant cultural role and reverence that the summit of Mauna Kea has always had within the indigenous Hawaiian community. We are most fortunate to have the opportunity to conduct observations from this mountain. This work is in part based on data obtained with the Hubble Space Telescope operated by AURA, Inc. for NASA under contract NAS5-26555 and with the Spitzer Space Telescope, operated by the Jet Propulsion Laboratory, California Institute of Technology under NASA contract 1407.

Facilities: Keck:I(MOSFIRE), HST(ACS/WFC3), Spit$z \operatorname{er}(\mathrm{IRAC})$.

\section{REFERENCES}

Ashby, M. L. N., Willner, S. P., Fazio, G. G., et al. 2013, ApJ, 769, 80 Bielby, R., Hudelot, P., McCracken, H. J., et al. 2012, A\&A, 545, A23 Bouwens, R. J., Illingworth, G. D., Oesch, P. A., et al. 2011, ApJ, 737, 90 Bouwens, R. J., Illingworth, G. D., Oesch, P. A., et al. 2015, ApJ, 803, 34 Bowler, R. A. A., Dunlop, J. S., McLure, R. J., et al. 2014, MNRAS, 440, 2810 Bradley, L. D., Trenti, M., Oesch, P. A., et al. 2012, ApJ, 760, 108 Caruana, J., Bunker, A. J., Wilkins, S. M., et al. 2014, MNRAS, 443, 2831 Faisst, A. L., Capak, P., Carollo, C. M., Scarlata, C., \& Scoville, N. 2014, ApJ, 788,87

Finkelstein, S. L., Papovich, C., Dickinson, M., et al. 2013, Natur, 502, 524

Finkelstein, S. L., Ryan, R. E., Jr., Papovich, C., et al. 2015, ApJ, arXiv: 1410.5439

Foreman-Mackey, D., Hogg, D. W., Lang, D., \& Goodman, J. 2013, PASP, 125,306

González, V., Bouwens, R., Illingworth, G., et al. 2014, ApJ, 781, 34 Grogin, N. A., Kocevski, D. D., Faber, S. M., et al. 2011, ApJS, 197, 35

Holden, B. P., Oesch, P. A., Gonzalez, V. G., et al. 2015, ApJ, arXiv: 1401.5490

Jiang, L., Bian, F., Fan, X., et al. 2013a, ApJL, 771, L6

Jiang, L., Egami, E., Mechtley, M., et al. 2013b, ApJ, 772, 99

Kashikawa, N., Shimasaku, K., Maukan, M. A., et al. 2006, ApJ, 648, 7

Kriek, M., Shapley, A. E., Reddy, N. A., et al. 2015, ApJS, in press (arXiv:1412.1835)

Labbé, I., Oesch, P. A., Bouwens, R. J., et al. 2013, ApJ, 777, L19

Laporte, N., Streblyanska, A., Clement, B., et al. 2014, A\&A, 562, L8

McLean, I. S., Steidel, C. C., Epps, H. W., et al. 2012, SPIE, 8446, 84460J

McLure, R. J., Dunlop, J. S., Bowler, R. A. A., et al. 2013, MNRAS, 432, 2696

Oesch, P. A., Bouwens, R. J., Illingworth, G. D., et al. 2012, ApJ, 745, 110

Oesch, P. A., Bouwens, R. J., Illingworth, G. D., et al. 2014, ApJ, 786, 108

Ono, Y., Ouchi, M., Mobasher, B., et al. 2012, ApJ, 744, 83

Ouchi, M., Shimasaku, K., Furusawa, H., et al. 2010, ApJ, 723, 869 
Pentericci, L., Fontana, A., Vanzella, E., et al. 2011, ApJ, 743, 132

Pentericci, L., Vanzella, E., Fontana, A., et al. 2014, ApJ, 793, 113

Planck Collaboration, Ade, P. A. R., Aghanim, N., et al. 2015, arXiv: 1502.01589

Salvaterra, R., Della Valle, M., Campana, S., et al. 2009, Natur, 461, 1258

Schaerer, D., \& de Barros, S. 2009, A\&A, 502, 423

Schenker, M. A., Ellis, R. S., Konidaris, N. P., \& Stark, D. P. 2014, ApJ, 795, 20

Schenker, M. A., Robertson, B. E., Ellis, R. S., et al. 2013, ApJ, 768, 196

Schenker, M. A., Stark, D. P., Ellis, R. S., et al. 2012, ApJ, 744, 179

Schmidt, K. B., Treu, T., Trenti, M., et al. 2014, ApJ, 786, 57

Shibuya, T., Kashikawa, N., Ota, K., et al. 2012, ApJ, 752, 114

Skelton, R. E., Whitaker, K. E., Momcheva, I. G., et al. 2014, ApJS, 214, 24

Smit, R., Bouwens, R. J., Franx, M., et al. 2015, ApJ, 801, 122
Smit, R., Bouwens, R. J., Labbé, I., et al. 2014, ApJ, 784, 58

Stark, D. P., Ellis, R. S., Chiu, K., Ouchi, M., \& Bunker, A. 2010, MNRAS, 408, 1628

Stark, D. P., Ellis, R. S., \& Ouchi, M. 2011, ApJL, 728, L2

Stark, D. P., Richard, J., Charlot, S., et al. 2015, arXiv:1408.3649

Stark, D. P., Schenker, M. A., Ellis, R., et al. 2013, ApJ, 763, 129

Tanvir, N. R., Fox, D. B., Levan, A. J., et al. 2009, Natur, 461, 1254

Tilvi, V., Papovich, C., Finkelstein, S. L., et al. 2014, ApJ, 794, 5

Trenti, M., Bradley, L. D., Stiavelli, M., et al. 2011, ApJ, 727, L39

Treu, T., Schmidt, K. B., Trenti, M., Bradley, L. D., \& Stiavelli, M. 2013 ApJL, 775, L29

Vanzella, E., Fontana, A., Pentericci, L., et al. 2014, A\&A, 569, A78

Vanzella, E., Pentericci, L., Fontana, A., et al. 2011, ApJL, 730, L35

Yan, H., Yan, L., Zamojski, M. A., et al. 2011, ApJL, 728, L22 